\title{
Effect of atomic layer deposition on the quality factor of silicon nanobeam cavities
}

\author{
Michael Gehl, ${ }^{1, *}$ Ricky Gibson, ${ }^{1}$ Joshua Hendrickson, ${ }^{2}$ Andrew Homyk, ${ }^{3}$ Antti Säynätjoki, ${ }^{4}$ Tapani \\ Alasaarela, ${ }^{4}$ Lasse Karvonen, ${ }^{4}$ Ari Tervonen, ${ }^{4}$ Seppo Honkanen, ${ }^{4}$ Sander Zandbergen, ${ }^{1}$ \\ Benjamin C. Richards, ${ }^{5}$ J. D. Olitzky, ${ }^{1}$ Axel Scherer, ${ }^{3}$ Galina Khitrova, ${ }^{1}$ \\ Hyatt M. Gibbs, ${ }^{1}$ Ju-Young Kim, ${ }^{6}$ and Yong-Hee Lee $^{6}$ \\ ${ }^{1}$ College of Optical Sciences, University of Arizona, 1630 East University Boulevard, Tucson, Arizona 85721, USA \\ ${ }^{2}$ Air Force Research Laboratory, Sensors Directorate, 2241 Avionics Circle, Building 600, \\ Wright Patterson Air Force Base, Ohio 45433, USA \\ ${ }^{3}$ Electrical Engineering and Kavli Nanoscience Institute, California Institute of Technology, \\ MC 200-36, 1200 East California Boulevard, Pasadena, California 91125, USA \\ ${ }^{4}$ Department of Micro- and Nanosciences, Aalto University School of Electrical Engineering, \\ P.O. Box 13500, 00076 Aalto, Finland \\ ${ }^{5}$ Advanced Concepts Team, Emcore Corporation, 10420 Research Road, Albuquerque, New Mexico 87123, USA \\ ${ }^{6}$ Department of Physics, Korea Advanced Institute of Science and Technology, Daejeon 305-701, Korea \\ ${ }^{*}$ Corresponding author: mgehl@optics.arizona.edu
}

Received October 4, 2011; revised December 12, 2011; accepted December 12, 2011; posted December 12, 2011 (Doc. ID 155915); published January 25, 2012

In this work we study the effect of thin-film deposition on the quality factor $(Q)$ of silicon nanobeam cavities. We observe an average increase in the $Q$ of $38 \pm 31 \%$ in one sample and investigate the dependence of this increase on the initial nanobeam hole sizes. We note that this process can be used to modify cavities that have larger than optimal hole sizes following fabrication. Additionally, the technique allows the tuning of the cavity mode wavelength and the incorporation of new materials, without significantly degrading $Q$. (c) 2012 Optical Society of America

OCIS codes: $\quad 350.4238,310.1860,310.6860$.

\section{MOTIVATION}

Photonic crystal (PC) cavities provide exciting platforms for photonics applications in addition to fundamental cavity quantum electrodynamics (cQED) experiments. The ability to fabricate cavities with small mode volumes $(V)$ and large $Q \mathrm{~s}$ leads to large enhancements in light-matter interactions. By placing active emitters such as quantum dots (QDs) into the cavity, it is easy to observe Purcell enhancement of spontaneous emission with only modest $Q$ s. Increasing the $Q$ further leads to the quantum regime of strong coupling, in which energy is able to coherently transfer between the emitter and cavity multiple times before decaying. This regime leads to exciting new physics, such as the Jaynes-Cummings ladder, deterministic single-photon sources, and photon blockades [1-3].

In addition to a high $Q$ and low $V$, it is important that the cavity and emitter be near resonance with each other. Previous work has shown the ability to reversibly tune cavity resonances by $5 \mathrm{~nm}$ using the condensation of gases at low temperatures [4]. Additionally, tuning of 2D photonic crystal systems has been shown using atomic layer deposition (ALD) of hafnium oxide [5] and titanium oxide [6] and plasmaenhanced chemical vapor deposition of silicon nitride [7]. In this work, we observe a shift in cavity resonance of 20 to $29 \mathrm{~nm}$, with a high tuning precision as a result of the multiplecycle deposition process.

Finally, it is important that one can incorporate an active medium with the cavity. While this can be achieved with
QDs and quantum wells in III-V systems, it presents more of a challenge for silicon photonics. In this work, we show the ability to deposit new material on top of a silicon PC cavity with a minimal effect on the $Q$. This opens up the possibility of incorporating active materials through similar methods. For example, one can dope $\mathrm{Al}_{2} \mathrm{O}_{3}$ with layers of erbium during the deposition process []․

\section{NANOBEAM CAVITIES}

One PC cavity geometry that is becoming popular is the $1 \mathrm{D}$ nanobeam, shown in Fig. 1. This design consists of a strip waveguide with an array of etched holes. The holes begin with uniform radius and spacing, creating a Bragg mirror region. The hole radius and spacing are then tapered down to a cavity region where the mode is confined, before tapering back up to another mirror region. The holes confine the light along one dimension, while it is confined by index guiding along the other two. The substrate can be etched from underneath the cavity, leaving it suspended, or in the case of this work, the cavity can remain on the substrate for increased durability. Simulations of unsuspended cavities predict $Q \mathrm{~s}$ as high as 366,000 with a mode volume of $0.552(\lambda / n)^{3}$. Experimentally, unsuspended silicon nanobeam cavities have already been measured with $Q$ s of 360,000 [9]].

In this work, nanobeams are fabricated on silicon-oninsulator wafers, consisting of a $220 \mathrm{~nm}$ silicon layer on top of a $2 \mu \mathrm{m} \mathrm{\textrm {SiO } _ { 2 }}$ layer. The structure is defined using electron beam lithography and formed using inductively coupled 


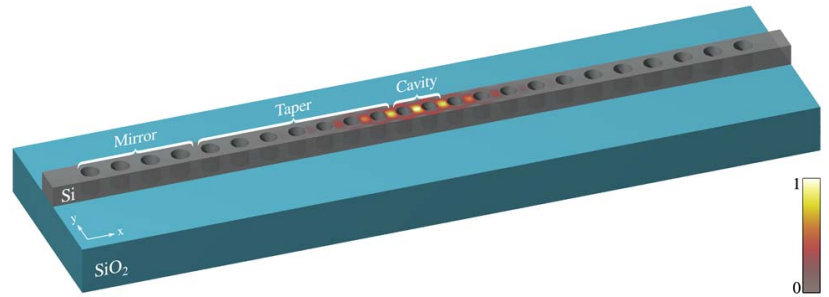

Fig. 1. (Color online) Schematic of the 1D nanobeam cavity with overlay of normalized electric field energy density.

plasma etching. Each nanobeam is characterized by a lattice constant " $a$," which is the distance between the center of the holes in the mirror region. The value of $a$ varies from 390 to $455 \mathrm{~nm}$ in $5 \mathrm{~nm}$ steps, shifting the cavity mode through the range of our measurement ability. In the taper region, the lattice constant varies from $0.98 a$ to $0.86 a$, while in the cavity region, the lattice constant is $0.84 a$. The nominal hole radius is 0.3 times the local lattice constant.

\section{CHARACTERIZATION BY MEASUREMENT OF THE CAVITY QUALITY FACTOR $Q$}

In order to characterize the $Q$ of silicon nanobeam cavities, we use the tapered microfiber probe technique [10,11]. In this technique, an optical fiber is heated and stretched, such that it adiabatically tapers to a region with a diameter on the order of $1 \mu \mathrm{m}$. This region of the fiber is then carefully positioned across the nanobeam cavity. A tunable narrowband laser source is coupled into one end of the fiber, and the transmitted power is detected at the other end. As the laser source is tuned into resonance with the cavity, some of the power is coupled into the cavity mode, resulting in a measurable dip in transmission through the fiber.

As a result of the tapered microfiber probe, the cavity experiences an additional amount of loss. This causes an increase in the measured resonance linewidth and thus a decrease in the measured $Q$ compared to the intrinsic $Q$ of the cavity. This effect was studied in [10], which found that by positioning the probe near the edge of the nanobeam, coupling to the cavity is minimized, leading to a measured $Q$ closer to the intrinsic $Q$. For this study, all $Q \mathrm{~s}$ are measured at the edge of the nanobeam cavity. Because of the positioning uncertainty of the probe, which leads to slight changes in the coupling and hence the measured $Q$, five measurements are taken of each cavity; the maximum value is used for all comparisons.
To determine the intrinsic $Q$ of a cavity, we use the crossed polarizer resonant scattering technique $[\underline{10,12}]$. This is a free space method in which a tunable laser source is focused onto the cavity with a microscope objective. The incident light is polarized at $45^{\circ}$ with respect to the polarization of the cavity mode. The reflected light is passed through a polarizer at $90^{\circ}$ relative to the incident light. Light that simply reflects from the substrate will be blocked by the crossed polarizer. Light that couples into the cavity will scatter out with the polarization of the cavity mode, a portion of which will be able to pass through the final polarizer and be detected. Because of the increased difficulty and low signal-to-noise ratio of this measurement, this technique is used only to determine the intrinsic $Q$ of the best cavities.

\section{ATOMIC LAYER DEPOSITION}

Atomic layer deposition is a thin-film deposition process that works through the sequential application of two or more gas phase chemicals. The growth takes place as a chemical reaction at the sample surfaces. The reaction is self-limiting, allowing accurate control of film thickness simply by counting the number of cycles.

Because of the nature of this deposition technique, the resulting film is conformal and grows along the surface normal [13]. This effect has already been utilized to grow microlens arrays [14] and planarized optical gratings [15] and to tune photonic crystal waveguides $[\underline{7,16}]$. The conformal film also reduces surface roughness, and $\mathrm{ALD}$-grown $\mathrm{Al}_{2} \mathrm{O}_{3}$ and $\mathrm{TiO}_{2}$ films have been found to significantly reduce propagation losses in silicon strip and slot waveguides [13,17]. Because the geometry of the silicon nanobeam cavity is a modified silicon strip waveguide, we believe that this process could lead to decreased losses and higher $Q \mathrm{~s}$ in these structures.

For this work, we investigated the thin-film growth of both $\mathrm{TiO}_{2}$ and $\mathrm{Al}_{2} \mathrm{O}_{3}$ on nanobeam cavities by $\mathrm{ALD}$. $\mathrm{TiO}_{2}$ is deposited at $120{ }^{\circ} \mathrm{C}$ using a process with titanium tetrachloride $\left(\mathrm{TiCl}_{4}\right)$ and water as precursors [18] $\mathrm{Al}_{2} \mathrm{O}_{3}$ is deposited at $200{ }^{\circ} \mathrm{C}$ using a process with trimethylaluminum $\left(\mathrm{Al}_{2}\left(\mathrm{CH}_{3}\right)_{6}\right)$ and water as precursors [19]. Figure $\underline{2}$ shows scanning electron micrograph (SEM) images of a cavity before and after the deposition of $20 \mathrm{~nm}$ of $\mathrm{Al}_{2} \mathrm{O}_{3}$, showing a clear reduction in hole size due to the thin film.

\section{EXPERIMENTAL RESULTS}

For initial tests, two nanobeam samples were fabricated using the same design parameters. They were first characterized using the tapered microfiber technique. While imaging the
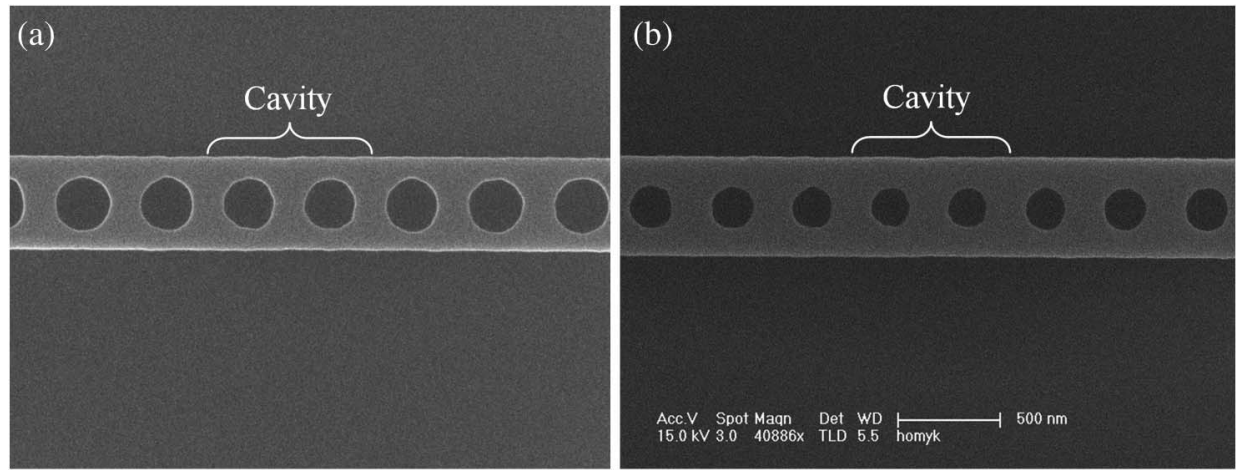

Fig. 2. SEM images of the cavity region of a silicon nanobeam (a) before and (b) after deposition of $20 \mathrm{~nm} \mathrm{Al}_{2} \mathrm{O}_{3}$ by $\mathrm{ALD}$. 

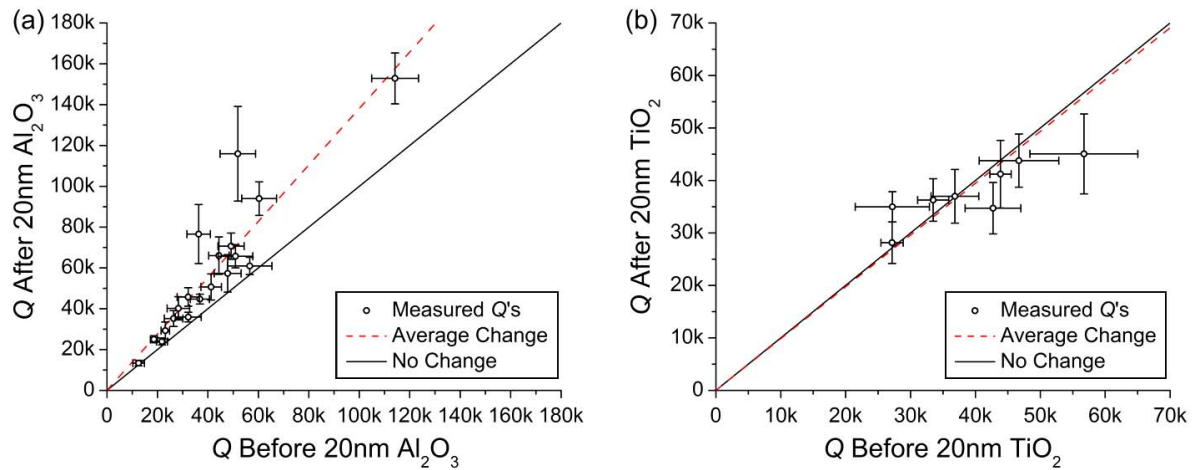

Fig. 3. (Color online) $Q$ of several cavities after ALD plotted against the $Q$ measured before $\mathrm{ALD}$, for $20 \mathrm{~nm}$ of (a) $\mathrm{Al}_{2} \mathrm{O}_{3}$ and (b) TiO $\mathrm{Tl}_{2}$. The solid line has a slope of 1 and represents no change in the $Q$, while the dotted line represents the average change of $38 \%$ for $\mathrm{Al}_{2} \mathrm{O}_{3}$ and $-1.3 \%$ for $\mathrm{TiO}_{2}$.

cavities, it was noticed that there was residual mask material remaining from fabrication. This material was removed using a 10 min $\mathrm{O}_{2}$ plasma etch. The cavities were recharacterized, and it was noted that the cavity wavelength decreased by $6.5 \mathrm{~nm}$ on average, but there was no significant change in cavity $Q$. One sample was then coated with $20 \mathrm{~nm}$ of $\mathrm{TiO}_{2}$ (index 2.27 at $\lambda=1.55 \mu \mathrm{m}$ ), while the other was coated with $20 \mathrm{~nm}$ of $\mathrm{Al}_{2} \mathrm{O}_{3}$ (index 1.62 at $\lambda=1.55 \mu \mathrm{m}$ ). The samples were characterized again, following the same procedure. The $\mathrm{TiO}_{2}\left(\mathrm{Al}_{2} \mathrm{O}_{3}\right)$ coated sample showed a shift in cavity mode of $59.2 \pm$ $0.7(30.3 \pm 0.8) \mathrm{nm}$ on average. The results of the $Q$ measurements are plotted in Fig. 3. While one $\mathrm{TiO}_{2}$ coated cavity showed an increase in $Q$ of $\overline{2} 9 \%$, the average change in $Q$ over all cavities was $-1.3 \pm 16 \%$. Every $\mathrm{Al}_{2} \mathrm{O}_{3}$ coated cavity showed an increase in $Q$, with an average increase of $38 \pm$ $31 \%$ and a maximum increase of $124 \%$. The large standard deviation for this sample mostly results from 2 of the 19 cavities, which showed an increase of more than $100 \%$. Figure 4 shows a measurement of a cavity from the $\mathrm{Al}_{2} \mathrm{O}_{3}$-coated sample before and after deposition. This measurement was performed using the crossed polarizer resonant scattering technique, so the measured $Q$ is that of the intrinsic $Q$. This shows the cavity $Q$ to have increased from 107,000 to 212,000 .

While the deposition of $20 \mathrm{~nm}$ of $\mathrm{Al}_{2} \mathrm{O}_{2}$ on that particular sample resulted in a clear increase in the cavity $Q$, from these initial tests it is not clear what factors contributed to this increase. The fact that the sample coated with $\mathrm{TiO}_{2}$ did not show the same increases suggests that the effect is not exclusively due to a decrease in surface roughness. Finitedifference time-domain (FDTD) simulations of the silicon nanobeam $Q$ [Fig. 5(a)] indicate that the $Q$ is highly dependent on the hole radius, with a peak $Q$ near a radius of 0.3 times the lattice constant. To account for the decrease in hole radius as a result of the ALD coating, a third sample was fabricated with an added offset to the hole radius. For each lattice constant, 13 cavities were defined with offsets ranging from -30 to $+30 \mathrm{~nm}$ in $5 \mathrm{~nm}$ steps. Four arrays of these cavities were fabricated with varying electron beam dosage, but only two arrays provided useful cavities.

This sample was characterized, coated with $20 \mathrm{~nm}$ of $\mathrm{Al}_{2} \mathrm{O}_{2}$, and then recharacterized. Fifty-three of the 60 cavities that were characterized on this third sample showed increases in the $Q$, with the average increase being $20 \pm 19 \%$. Figure 5(b) shows a plot of the measured $Q$ prior to ALD coating as a function of hole radius offset. There is a trend toward higher $Q \mathrm{~s}$ for cavities with smaller holes. Unlike the simulation, there is not a clear peak in the $Q$. This suggests that our range of hole sizes did not cover the designed range. Looking at the percent increase in the $Q$ as a function of the hole radius offset (Fig. 6), there is also a clear trend toward a greater increase for cavities with larger holes. Following ALD, these cavities have hole sizes closer to those of the cavities that showed the highest $Q \mathrm{~s}$ prior to ALD. This is consistent with the idea that the increase in $Q$ is due to the shift of hole size closer to the optimal (highest $Q$ ) design.

Figure 7 shows a plot of the shift in the cavity mode wavelength as $\bar{a}$ result of the deposition of $20 \mathrm{~nm}$ of $\mathrm{Al}_{2} \mathrm{O}_{3}$, along with FDTD simulation results. While the wavelength shift showed very little dependence on the lattice constant, it showed a strong dependence on the initial hole radius offset.
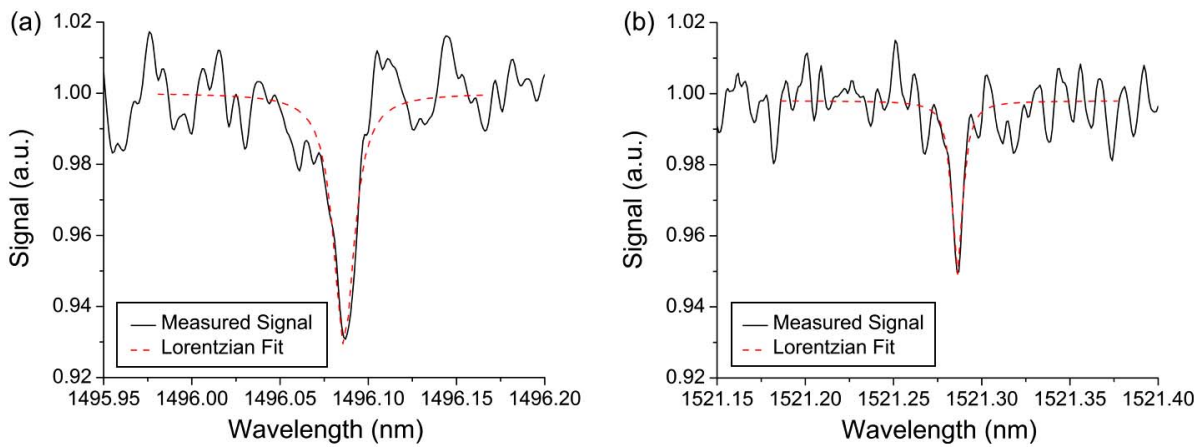

Fig. 4. (Color online) Crossed polarizer resonant scattering measurement of a cavity (a) before and (b) after being coated with $20 \mathrm{~nm}$ of $\mathrm{Al}_{2} \mathrm{O}_{3}$, yielding a measured $Q$ of 107,000 before and 212,000 after. The before data were collected prior to the $\mathrm{O}_{2}$ plasma etch, which removed the mask residue and shifted the cavity peak to shorter wavelength by $6.5 \mathrm{~nm}$. 

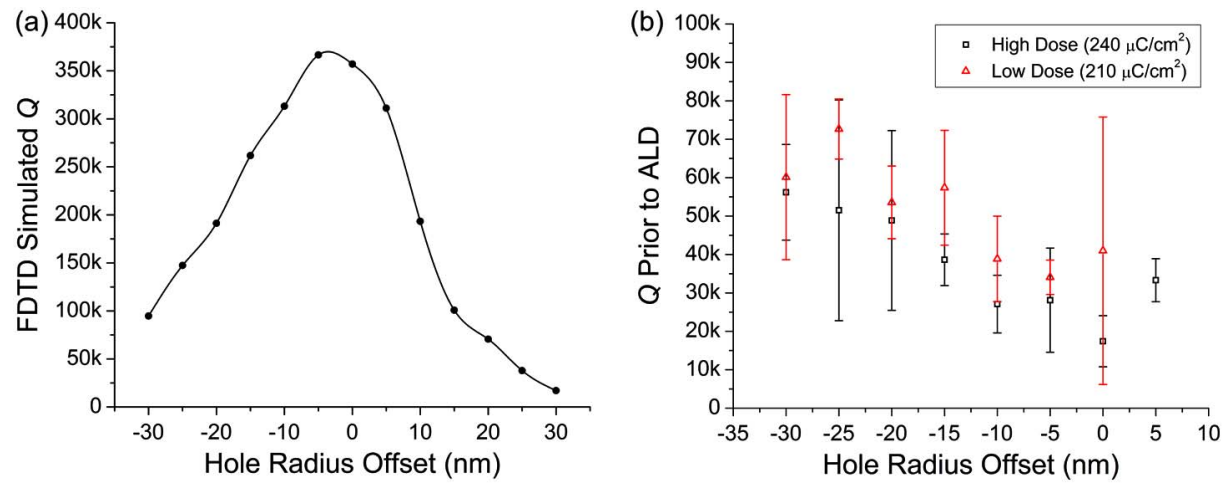

Fig. 5. (Color online) (a) FDTD simulation of the $Q$ prior to ALD coating, showing the expected peak around $0 \mathrm{~nm}$ offset. The $0 \mathrm{~nm}$ offset corresponds to a hole radius of 0.3 times the lattice constant. (b) Plot of the average measured $Q$ of several cavities prior to ALD coating versus the hole radius offset, showing a trend of higher $Q$ for cavities with smaller holes (more negative offset). Dose refers to the dosage used during electron beam lithography.

The shift also showed some dependence on the dosage used during electron beam lithography. This is likely due to the dependence of the hole size and beam width on electron beam dose. The higher dose array, which should have larger holes due to the increased exposure of the mask, showed wavelength shifts in the range of $21.6 \pm 0.2 \mathrm{~nm}$ for the smallest holes to $28.0 \pm 0.1 \mathrm{~nm}$ for the largest holes. The lower dose array showed wavelength shifts in the range of $20.2 \pm$ $0.2 \mathrm{~nm}$ for the smallest holes to $27.1 \pm 0.4 \mathrm{~nm}$ for the largest. The FDTD simulations predict a similar range of wavelength shift. The tuning precision is very high because it is

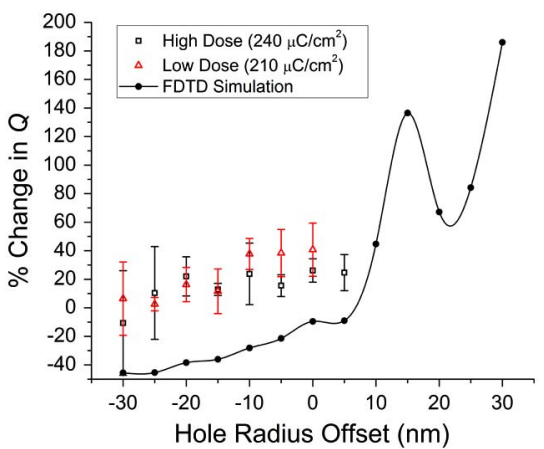

Fig. 6. (Color online) Plot of the average percent change in the $Q$ of the cavities from Fig. 4 versus the initial hole radius offset, showing a trend of greater increase for cavities that began with larger holes (more positive offset). The FDTD results are plotted for comparison.

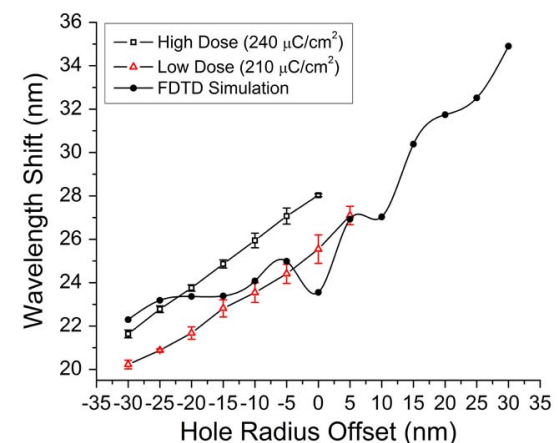

Fig. 7. (Color online) Plot of the average shift in wavelength of the cavity mode following ALD versus the hole radius offset, along with the FDTD simulation results. determined by the growth rate of the thin film, which can be very slow. For the ALD technique used in this work, the growth rate was around $0.1 \mathrm{~nm} /$ cycle, with a cycle time of $3.45 \mathrm{~s}$. This results in a wavelength shift on the order of $0.1-0.15 \mathrm{~nm} /$ cycle.

In order to investigate the effect of the $\mathrm{Al}_{2} \mathrm{O}_{3}$ coating on the mode volume, we use numerical methods. FDTD simulations reveal a nearly linear relationship between the mode volume and hole radius offset. The mode volume increases from $0.410(\lambda / n)^{3}$ for a $+30 \mathrm{~nm}$ offset, to $0.658(\lambda / n)^{3}$ for $\mathrm{a}-30 \mathrm{~nm}$ offset. At the same time, the increase in mode volume as a result of depositing $20 \mathrm{~nm}$ of $\mathrm{Al}_{2} \mathrm{O}_{3}$ shows little dependence on the hole radius offset, fluctuating in the range of a $2.06 \%$ to $6.45 \%$ increase.

\section{CONCLUSIONS}

We have shown that ALD can be a valuable tool for tuning the resonant wavelength and $Q$ of $1 \mathrm{D}$ silicon nanobeam cavities postfabrication. The low index and low deposition rate of $\mathrm{Al}_{2} \mathrm{O}_{3}$ allows for very precise control of the wavelength shift. The initial size of the cavity holes also plays an important role in the resonance shift. Cavities with smaller holes to begin with will have modes that are more confined to the silicon and therefore will see less of an effect from the additional dielectric material.

Initial results show that a $20 \mathrm{~nm}$ coating of $\mathrm{Al}_{2} \mathrm{O}_{3}$ can increase the $Q$ of $1 \mathrm{D}$ silicon nanobeam cavities by $38 \pm 31 \%$. While the exact nature of this change in the $Q$ is not fully understood, both simulation and experiment show that the $Q$ is significantly affected by the size of the cavity holes. Therefore, it is likely that the changing hole size resulting from the $\mathrm{Al}_{2} \mathrm{O}_{3}$ deposition is responsible for much of the change in the $Q$ observed in this work. There still remains much to be done to determine the contribution of other effects to the change in the $Q$. The conformal nature of the ALD film will lead to decreased surface roughness and decreased scattering losses at the $\mathrm{Al}_{2} \mathrm{O}_{3}$-air interface; however, the large index mismatch of $\mathrm{Al}_{2} \mathrm{O}_{3}$ and silicon means that scattering at this interface will still be significant. Additionally, the thin film could be causing surface passivation, leading to decreased absorption by surface states. Finally, the film may be affecting the mode profile, pulling the field away from the oxide layer and decreasing losses into the substrate. Repeating these experiments with 
various materials and film thicknesses will reveal more detail on these separate effects.

In summary, the ability to deposit $\mathrm{Al}_{2} \mathrm{O}_{3}$ onto silicon nanobeams without significantly degrading the $Q$ or increasing $V$ is important for the field of silicon photonics and cQED. In addition to being able to tune the cavity resonance, the ability to incorporate new materials, such as erbium, will allow the fabrication of active devices from silicon PC cavities.

\section{ACKNOWLEDGMENTS}

M. Gehl acknowledges support by the Department of Defense (DoD) through the National Defense Science \& Engineering Graduate Fellowship (NDSEG) program. S. Zandbergen acknowledges partial support from Arizona Technology \& Research Initiative Funding (TRIF). J. Hendrickson acknowledges support from the Air Force Office of Scientific Research (AFOSR)-LRIR 10RY04COR (Gernot Pomrenke, program manager). A. Homyk appreciates the generous support of the ARCS Foundation. A. Säynätjoki was supported by Academy of Finland grant 134087 and a travel grant by the Finnish Foundation for Technology Promotion. The Caltech and UofA groups thank NSF ERC CIAN (EEC-0812072) for support. The UofA group acknowledges support from the AFOSR (FA955010-1-0003) and NSF EPMD (ECCS-1101341).

\section{REFERENCES}

1. P. R. Berman, Cavity Quantum Electrodynamics (Academic, 1994).

2. K. Vahala, Optical Microcavities (World Scientific, 2004).

3. G. Khitrova, H. M. Gibbs, M. Kira, S. W. Koch, and A. Scherer, "Vacuum Rabi splitting in semiconductors," Nat. Phys. 2, 81-90 (2006).

4. S. Moser, J. Hendrickson, B. C. Richards, J. Sweet, G. Khitrova, H. M. Gibbs, T. Yoshie, A. Scherer, O. B. Shchekin, and D. G. Deppe, "Scanning a photonic crystal slab nanocavity by condensation of xenon," Appl. Phys. Lett. 87, 052101 (2005).

5. X. Yang, C. J. Chen, C. A. Husko, and C. W. Wong, "Digital resonance tuning of high- $Q / V_{m}$ silicon photonic crystal nanocavities by atomic layer deposition,” Appl. Phys. Lett. 91, 161114 (2007).

6. S. Schartner, S. Kalchmair, A. M. Andrews, P. Klang, W. Schrenk and G. Strasser, "Post-fabrication fine-tuning of photonic crystal quantum well infrared photodetectors,” Appl. Phys. Lett. 94, 231117 (2009).

7. E. Graugnard, D. P. Gaillot, S. N. Dunham, C. W. Neff, T. Yamashita, and C. J. Summers, "Photonic band tuning in two- dimensional photonic crystal slab waveguides by atomic layer deposition,” Appl. Phys. Lett. 89, 181108 (2006).

8. K. Solehmainen, M. Kapulainen, P. Heimala, and K. Polamo, "Erbium-doped waveguides fabricated with atomic layer deposition method," IEEE Photon. Technol. Lett. 16, 194-196 (2004).

9. E. Kuramochi, H. Taniyama, T. Tanabe, K. Kawasaki, Y.-G. Roh, and M. Notomi, "Ultrahigh- $Q$ one-dimensional photonic crystal nanocavities with modulated mode-gap barriers on $\mathrm{SiO}_{2}$ claddings and on air claddings," Opt. Express 18, 15859-15869 (2010).

10. B. C. Richards, J. Hendrickson, J. D. Olitzky, R. Gibson, M. Gehl, K. Kieu, U. K. Khankhoje, A. Homyk, A. Scherer, J.-Y. Kim, Y.-H. Lee, G. Khitrova, and H. M. Gibbs, "Characterization of 1D photonic crystal nanobeam cavities using curved microfiber," Opt. Express 18, 20558-20564 (2010).

11. I.-K. Hwang, S.-K. Kim, J.-K. Yang, S.-H. Kim, S.-H. Lee, and Y.-H. Lee, "Curved-microfiber photon coupling for photonic crystal light emitter,” Appl. Phys. Lett. 87, 131107 (2005).

12. M. W. McCutcheon, G. W. Rieger, I. W. Cheung, J. F. Young, D. Dalacu, S. Frédérick, P. J. Poole, G. C. Aers, and R. L. Williams, "Resonant scattering and second-harmonic spectroscopy of planar photonic crystal microcavities,” Appl. Phys. Lett. 87, 221110 (2005).

13. T. Alasaarela, D. Korn, L. Alloatti, A. Säynätjoki, A. Tervonen, R. Palmer, J. Leuthold, W. Freude, and S. Honkanen, "Reduced propagation loss in silicon strip and slot waveguides coated by atomic layer deposition,” Opt. Express 19, 11529-11538 (2011).

14. J. J. Wang, A. Nikolov, and Q. Wu, "Nano- and microlens arrays grown using atomic-layer deposition," IEEE Photon. Technol. Lett. 18, 2650-2652 (2006).

15. J. J. Wang, X. Deng, R. Varghese, and A. Nikolov, "Filling high aspect-ratio nano-structures by atomic layer deposition and its applications in nano-optic devices and integrations," J. Vac. Sci. 23, 3209-3213 (2005).

16. D. Gaillot, E. Graugnard, J. Blair, and C. Summers, "Dispersion control in two-dimensional superlattice photonic crystal slab waveguides by atomic layer deposition," Appl. Phys. Lett. 91, 181123 (2007).

17. A. Säynätjoki, L. Karvonen, T. Alasaarela, X. Tu, T. Y. Liow, M. Hiltunen, A. Tervonen, G. Q. Lo, and S. Honkanen, "Low-loss silicon slot waveguides and couplers fabricated with optical lithography and atomic layer deposition," Opt. Express 19 26275-26282 (2011).

18. T. Alasaarela, T. Saastamoinen, J. Hiltunen, A. Säynätjoki, A. Tervonen, P. Stenberg, M. Kuittinen, and S. Honkanen, "Atomic layer deposited titanium dioxide and its application in resonant waveguide grating," Appl. Opt. 49, 4321-4325 (2010).

19. R. L. Puurunen, "Surface chemistry of atomic layer deposition: A case study for the trimethylaluminum/water process," J. Appl. Phys. 97, 121301 (2005). 